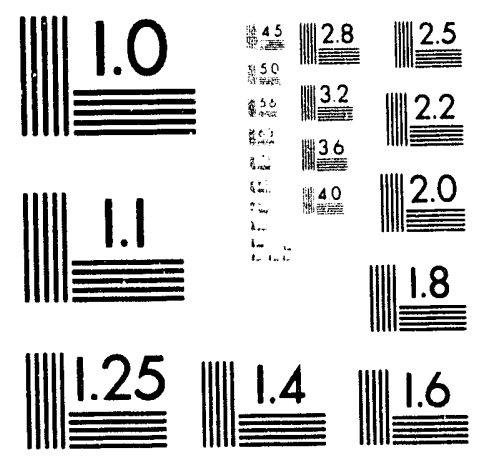



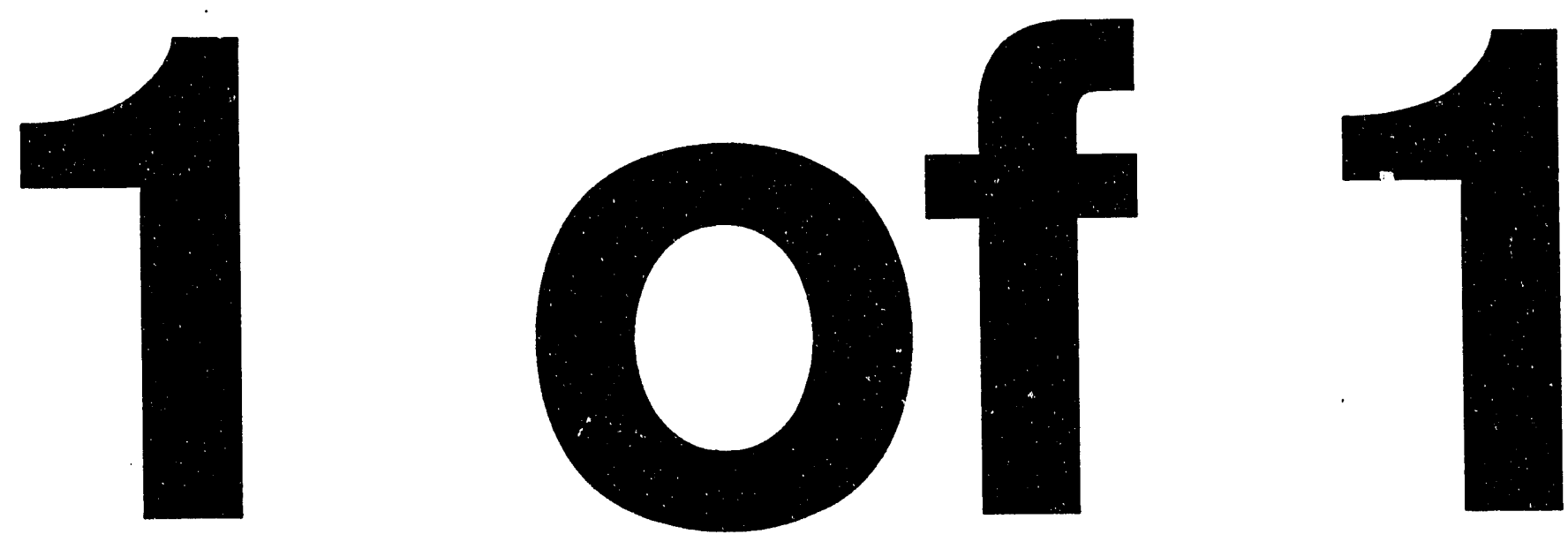


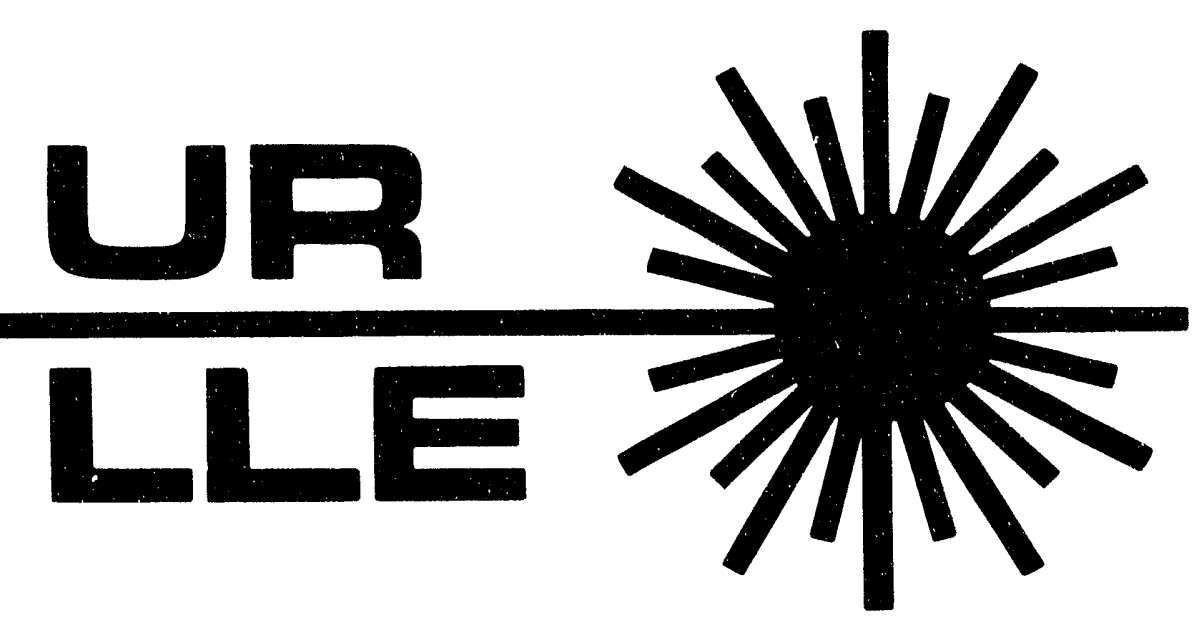

\title{
STABILITY ANALYSIS OF UNSTEADY ABLATION FRONTS
}

\section{by}

\author{
R. Betti, R. L. McCrory, and C. P. Verdon
}

August 1993

Laboratory Report No. 238

\section{DISCLAIMER}

This report was prepared as an account of work sponsored by an agency of the United States Government. Neither the United States Government nor any agency thereof, nor any of their employees, makes any warranty, express or implied, or assumes any legal liability or responsibility for the accuracy, completeness, or usefulness of any information, apparatus, product, or process disclosed, or represents that its use would not infringe privately owned rights. Reference herein to any specific commercial product, process, or service by trade name, trademark. manufacturer, or otherwise does not necessarily constitute or imply its endorsement, recommendation, or favoring by the United States Government or any agency thereof. Tile views and opinions of authors expressed herein do not necessarily state or reflect those of the United States Government or any agency thereof.

\section{Laboratory for Laser Energetics}

\section{College of Engineering and Applied Science University of Rochester}

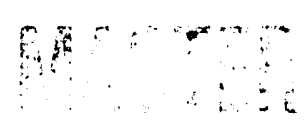




\title{
STABILITY ANALYSIS OF UNSTEADY ABLATION FRONTS \\ R. Betti, R. L. McCrory, and C. P. Verdon \\ LABORATORY FOR LASER ENERGETICS \\ University of Rochester \\ 250 East River Road \\ Rochester, NY 14623-1299

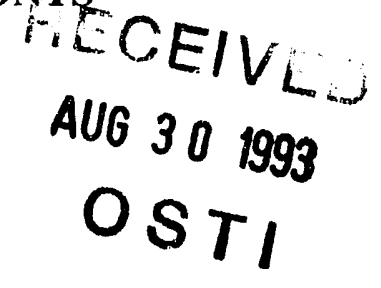

\begin{abstract}
The linear stability analysis of unsteady ablation fronts, is carried out for a semiinfinite uniform medium. For a laser accelerated target, it is shown that a properly selected modulation of the laser intensity can lead to the dynamic stabilization or growthrate reduction of a large portion of the unstable spectrum. The theory is in qualitative agreement with the numerical results obtained by using the two-dimensional hydrodynamic code $O R C H I D$.
\end{abstract}

The classical Rayleigh-Taylor instability 1 occurs when a heavy fluid is accelerated by a lighter fluid. In inertial confinement fusion (ICF) the heavy fluid is the compressed ablated target material that is accelerated by the low-density ablated plasma. The classical treatment of the incompressible Rayleigh-Taylor instability leads to a linear growth rate given by $\gamma=\sqrt{|k g| A}$, where $k$ is the instability wave number, $g$ the acceleration, and $A$ the Atwood number $A=\left(\rho_{h}-\rho_{l}\right) /\left(\rho_{h}+\rho_{l}\right)\left(\rho_{l}\right.$ and $\rho_{h}$ represent the light and heavy fluid densities, respectively). For typical (ICF) parameters, a classical Rayleigh-Taylor instability would produce an unacceptably large amount of distortion in the unablated target resulting in a degraded capsule performance with respect to final core conditions. Thus, it is of great importance to study the possible means for suppression of the ablation surface instability in ICF. It has been recently shown that the ablation process leads to convection of the perturbation away from the interface between the two fluids. ${ }^{2--5}$ Since the instability is localized at the interface, the ablative convection stabilizes short wavelength modes. The typical growth rate of the ablative RayleighTaylor instability can be written in the following approximate form: ${ }^{3}$

$$
\gamma=\sqrt{|k g| A}-\beta\left|k V_{a}\right|,
$$

where $V_{a}$ is the ablation velocity and $\beta$ is a numerical factor $(\beta \simeq 3-4)$. 
In this paper we show that a properly selected modulation of the laser intensity can significantly reduce the unstable spectrum and the maximum growth rate. To treat the analytic linear stability of unsteady ablation fronts, we consider a simplified sharp boundary model consisting of a heavy fluid, with density $\rho_{h}$, superimposed to a lighter fluid $\left(\rho_{l}\right)$, in the force field $\mathbf{g}(t)=g(t) \mathbf{e}_{y}$ opposite to the density gradient $\left[g(t)<0\right.$ and $\mathbf{e}_{y}$ is the unit vector in the direction of the density gradient] and with arbitrary time dependence. The heavy fluid is moving downward with velocity $\mathbf{U}_{h}=-V_{a} \mathbf{e}_{y}$ and the lighter fluid is ejected with velocity $U_{l}$. The equilibrium velocities $U_{l}(t)$ and $U_{h}(t)$ are both dependent on the ablation ratio per unit surface $\dot{m}(t)$, that is treated as an arbitrary function of time. The equilibrium can be readily derived from conservation of mass and momentum. We consider a class of equilibria with nonuniformities localized at the interface between the two fluids. Continuity of the mass flow and the pressure balance across the interface lead to the following conditions:

$$
\begin{gathered}
\rho_{l} U_{l}(t)=\rho_{h} U_{h}(t) \\
P_{h}-P_{l}=\rho_{l} U_{l}^{2}(t)-\rho_{h} U_{h}^{2}(t),
\end{gathered}
$$

where $P_{h}$ and $P_{l}$ represent the pressure of the heavy and light fluid at the interface. Notice that $U_{l / h}<0$ in the chosen frame of reference. We assume that the discontinuities in the equilibrium quantities can be removed by including the physics of the ablation process.

The linear stability problem can be greatly simplified by an appropriate choice of the linearized equation of state. It is widely known that the most Rayleigh-Taylor unstable perturbations are incompressible. Furthermore, the ablative stabilization is a convective process and, therefore, independent of the equation of state. It follows that the essential physics of the instability can be captured by a simple incompressible flow model. The stability analysis proceeds in a standard manner. All perturbed quantities are written as $Q_{1}=\tilde{Q}(y, t) \exp (i k x)$ and the system of equations describing the linear evolution of the perturbation assumes the following form:

$$
\begin{gathered}
\left(\partial_{t}+U_{j} \partial_{y}\right) \tilde{\rho}_{j}=0, \\
\rho_{j}\left(\partial_{t}+U_{j} \partial_{y}\right) \tilde{v}_{j x}=-i k \tilde{p}_{j} \\
\rho_{j}\left(\partial_{t}+U_{j} \partial_{y}\right) \tilde{v}_{j y}+\tilde{\rho}_{j} \partial_{t} U_{j}=-\partial_{y} \tilde{p}_{j}+\tilde{\rho}_{j} g \\
i k \tilde{v}_{j x}+\partial_{y} \tilde{v}_{j y}=0 .
\end{gathered}
$$


where the subscript $j$ denotes the heavy fluid region $(j=h)$ and the light fluid region $(j=l)$ and $\partial_{y}=\partial / \partial y, \partial_{t}=\partial / \partial t$. The two regions are separated by an interface (the ablation front), that moves with the heavy fluid. The linear displacement of the interface $\tilde{\eta}(t) \exp (i k x)$, has to account for the heavy fluid ablative convection and can be described by the following integral equation:

$$
\tilde{\eta}(t)=\int_{-\infty}^{t} \tilde{v}_{h y}\left[y_{0}\left(t^{\prime}\right), t^{\prime}\right] d t^{\prime}
$$

where $y_{0}\left(t^{\prime}\right)=\int_{t}^{t^{\prime}} U_{h}\left(t^{\prime \prime}\right) d t^{\prime \prime}$ is the unperturbed trajectory of the fluid element that, at time $t$, has reached the ablation front. Since the heavy and light fluid extend to infinity and the instability is expected to be localized at the interface, the perturbation must vanish at $y \rightarrow \pm \infty$. A set of jump conditions relating the values of the physical quantities in the two regions can be derived from the incompressibility and the conservation equations. At the interface $(y=0)$, the set of jump conditions can be written in the following form:

$$
\begin{gathered}
\tilde{v}_{h y}=\tilde{v}_{l y} \\
\left(\rho_{h}-\rho_{l}\right)\left(\partial_{t} \tilde{\eta}-\tilde{v}_{h y}\right)-U_{h} \tilde{\rho}_{h}+U_{l} \tilde{\rho}_{l}=0 \\
\tilde{v}_{h x}-\tilde{v}_{l x}+i k \tilde{\eta}\left(U_{h}-U_{l}\right)=0 \\
\tilde{p}_{h}-\tilde{p}_{l}+\tilde{\rho}_{h} U_{h}^{2}-\tilde{\rho}_{l} U_{l}^{2}+g\left(\rho_{h}-\rho_{l}\right) \tilde{\eta}=0 .
\end{gathered}
$$

The next step is to sclve Eq. (4) in the two regions and then apply the jump conditions.

The solution of the linearized equation in the heavy fluid region $(h)$ is greatly simplified by the following transformation of variable: $y_{h}=y-\int^{t} U_{h}\left(t^{\prime}\right) d t^{\prime}$. A straightforward calculation leads to the following form of the perturbed variables in region $h$ :

$$
\begin{gathered}
\tilde{v}_{h y}=\tilde{v}_{h}(t) \exp \left(-k y_{h}\right)+\tilde{a}\left(y_{h}\right) \\
\tilde{v}_{h x}=\frac{i}{k} \frac{\partial \tilde{v}_{h y}}{\partial y_{h}} \\
\tilde{\rho}_{h}=\tilde{\rho}_{h}\left(y_{h}\right) \\
\tilde{p}_{h}=-\frac{\rho_{h}}{k^{2}} \frac{\partial^{2} \tilde{v}_{h y}}{\partial t \partial y_{h}}
\end{gathered}
$$


where $\tilde{u}_{h}(t), \tilde{\rho}_{h}\left(y_{h}\right)$, and $\tilde{a}\left(y_{h}\right)$ are arbitrary functions of $t$ and $y_{h}$, and $k$ is chosen to be positive $(k>0)$. In order to satisfy the boundary conditions, $\tilde{a}$ and $\tilde{\rho}_{h}$ have to vanish at $y_{h} \rightarrow \infty$. Since $\lim _{t \rightarrow \infty} y_{h}=\infty$, it follows that $\tilde{a}$ and $\tilde{\rho}_{h}$ asymptotically vanish in time. In our asymptotic stability analysis, we neglect all the quantities that do not grow in time. Thus, we set $\tilde{a}=0$ and $\tilde{\rho}_{h}=0$.

We apply the same procedure to the light fluid region $(l)$ and define the new coordinates $y_{l}=y-\int^{t} U_{l}\left(t^{\prime}\right) d t^{\prime}$. The solution of the linearized equations in region $l$ can be written in the following form:

$$
\begin{gathered}
\tilde{v}_{l y}=\tilde{u}_{l}(t) \exp \left(k y_{l}\right)+\tilde{b}\left(y_{l}\right)+\tilde{c}\left(y_{l}\right) f(t) \\
\tilde{v}_{l x}=\frac{i}{k} \frac{\partial \tilde{v}_{l y}}{\partial y_{l}} \\
\tilde{\rho}_{l}=\tilde{\rho}_{l}\left(y_{l}\right) \\
\tilde{p}_{l}=-\frac{\rho_{l}}{k^{2}} \frac{\partial^{2} \tilde{v}_{l y}}{\partial t \partial y_{l}}
\end{gathered}
$$

where $\tilde{b}\left(y_{l}\right)$ and $\tilde{\rho}_{l}\left(y_{l}\right)$ are free functions of $y_{l}$, that vanish at $y_{l} \rightarrow-\infty$, and $\tilde{u}_{l}(t)$ is an arbitrary function of $t$. The functions $\tilde{c}\left(y_{l}\right)$ and $f(t)$ satisfy the following differential equations:

$$
\begin{gathered}
{\left[\frac{d^{2}}{d y_{l}^{2}}-k^{2}\right] \tilde{c}+k^{2} \frac{\tilde{\rho}_{l}}{\rho_{l}}=0} \\
\frac{d f}{d t}=G(t),
\end{gathered}
$$

where

$$
G(t) \equiv g(t)-\frac{\partial U_{l}}{\partial t}
$$

The next step is to recognize that, using Eqs. (7) into Eq. (8), the interface equation can be rewritten in a differential form: $\left(\partial_{t}-k U_{h}\right) \tilde{\eta}=\tilde{v}_{h y}(y=0, t)$. 
After substituting Eqs. (7) and (8) into the jump conditions [Eqs. (6)] and using the differential form of the interface equation, the following ordinary differential equation for $\tilde{\eta}(t)$ is derived:

$$
\begin{aligned}
\left(\partial_{t}-k U_{l}\right) G^{-1} & \left\{\left(\partial_{t}-k U_{l}\right)\left(\partial_{t}-k U_{h}\right) \tilde{\eta}+\right. \\
& \left.A\left[k U_{l}\left(\partial_{t}-k U_{h}\right)+k g\right] \tilde{\eta}\right\}-A k^{2} U_{h} \tilde{\eta}=0,
\end{aligned}
$$

where $A \equiv\left(\rho_{h}-\rho_{l}\right) /\left(\rho_{h}+\rho_{l}\right)$ is the Atwood number. For ICF applications, the appropriate ordering $U_{h} / U_{l}=\rho_{l} / \rho_{h} \sim(1-A)<<1$ and $g>\partial U_{l} / \partial t$. To lowest order in $1-\mathrm{A}$, the last term in Eq. (11) can be neglected, yielding

$$
\left\{\left(\partial_{t}-k U_{l}\right)\left(\partial_{t}-k U_{h}\right)+A\left[k U_{l}\left(\partial_{t}-k U_{h}\right)+k g\right]\right\} \tilde{\eta}=0
$$

Equation (12) can be further simplified by using the Ansatz

$$
\tilde{\eta}(t)=\xi(t) \exp \left[\frac{3}{2} k \int^{t} U_{h}\left(t^{\prime}\right) d t^{\prime}\right]
$$

and by neglecting other terms of order $(1-A)<<1$. After some straightforward manipulations, we obtain

$$
\frac{d^{2}}{d t^{2}}+k\left[A g-\frac{1}{2} \frac{d V_{a}}{d t}-\frac{1}{4} k V_{a}^{2}\right] \xi=0
$$

where $g$, and $V_{a}$ are functions of time. Observe that, for steady equilibrium configurations, Eq. (13) and (14) yield the normal mode solution for $\tilde{\eta} \sim \exp (\gamma t)$, with $\gamma$ satisfying the dispersion relation

$$
\gamma=\sqrt{(|k g| A)+\frac{1}{4} k^{2} V_{a}^{2}}-\frac{3}{2}\left|k V_{a}\right|
$$

It is easy to recognize that the contribution of the second term under the square root is relevant only at very small wavelengths, where the mode is already strongly stabilized by convection [first term in Eq. (15)]. Neglecting such a term in Eq. (14) and (15), would only cause a small shift of the cut-off wave number $\left[\Delta k_{c} / k_{c}=1 / 9\right]$ that is consistent with the order of magnitude of the previous approximations. After neglecting such a term, Eq. (15) reproduces the numerically derived growth rate of Ref. (3) with $\beta=1.5$. Equations (13) and (14), which are valid for arbitrary unsteady configurations, are now applied to the particular equilibrium obtained by temporally modulating the laser 
intensity. Consider a planar target of thickness $d$ and density $\rho_{0}$ irradiated by a uniform laser beam. The periodically modulated laser intensity $\left[I(t)=I_{0}\left(1+\Delta \sin \omega_{0} t\right), \Delta \leq 1\right]$, induces an oscillating ablation pressure $P_{a}(t)=P_{0}\left(1+\Delta_{p} \sin \omega_{0} t\right)$ and ablation velocity $V_{a}(t)=V_{a 0}\left(1+\Delta_{a} \sin \omega_{0} t\right)$ with $\Delta_{p} \leqslant \Delta$ and $\Delta_{a} \leqslant \Delta$. For simplicity, we assume that the ablation pressure and the ablation velocity are directly proportional to the laser intensity and the ablation process develops on a very slow time scale compared to oscillation period and the sound transit time through the target $\left[V_{a}<<c_{s}, c_{s}\right.$ is the sound speed]. Although the scaling $V_{a} \sim \sqrt{I} \sim\left[1+\Delta_{a} \sin \left(\omega_{0} t\right)\right]^{1 / 2}$ is more appropriate than a simple linear dependence, the numerical simulations show that the ablation velocity is almost insensitive to the oscillations in the laser intensity $\left(\Delta_{a}<<1\right)$ and $V_{a} \simeq V_{a 0} . I_{0}$ and $P_{0}$ are two slowly varying functions of time $\left[V_{a} / d<\left(1 / I_{0}\right)\left(d I_{0} / d t\right)\right.$ $=\left(1 / P_{0}\right)\left(d P_{0} / d t\right)<<\omega_{0} \sim c_{s} / d j$. A simple estimate of the acceleration of the ablation front can be derived by solving the one-dimensional compressible fluid equations of Ref. [6], for a target accelerated by the ablation pressure. The result is

$$
g(t)=-\frac{d V_{a}}{d t}-L^{-1}\left\{\operatorname{coth}\left[\frac{s}{c_{s}}\left(d-\bar{y}_{a}\right)\right] \frac{s \hat{p}_{a}(s)}{\rho_{0} c_{s}}\right\}
$$

where $L^{-1}$ denotes the inverse Laplace transform, $s$ is the Laplace variable, and $\hat{p}_{a}(s)$ is the Laplace transform of the ablation pressure. The quantity $\bar{y}_{a}=\int_{0}^{t} V_{a}\left(t^{\prime}\right) d t^{\prime}$ is the position of the ablation front in the Lagrangian frame of the moving target. In deriving Eq. (16), the slow ablation time scale $\left(\sim d / V_{a}\right)$ has been treated as an independent variable. A simple expression for $g(t)$ can be derived in the asymptotic limit $\left(d / V_{a}\right)>t>>\left(d / c_{s}\right)$, yielding

$$
g(t)=-g_{0}\left[1+\alpha \sin \omega_{0} t+\epsilon \cos \omega_{0} t\right]
$$

where $\quad g_{0} \equiv P_{0} / \rho_{0} d_{a}, \quad \alpha \equiv \Delta_{p}\left(\omega_{0} d_{a} / c_{s}\right) \cot \left(\omega_{0} d_{a} / c_{s}\right)$, and $\quad \epsilon=V_{a 0} \Delta_{a} \omega_{0} / g_{0}$, $d_{a}=d-\bar{y}_{a}$. A more accurate estimate of $g(t)$ (and the parameters $g_{0}, \alpha$, and $\in$ ), can be obtained by using a one-dimensional code. Later in this letter, we use the onedimensional hydrodynamic code $L I L A C^{8}$ to derive $g_{0}, \alpha$, and $\in$. However, Eq. (17) gives some physical insight of the relevant quantities that affect the oscillation amplitude in the target acceleration. In particular, large oscillations can be achieved by values of the modulation period shorter than the sound transit time through the target $\left[T_{0} \equiv 2 \pi / \omega_{0}<d / c_{s}\right]$. Before proceeding further, it is important to define the range of validity of the stability model for the prescribed equilibrium. The oscillations in the ablation pressure propagates inside the target at the sound speed. Thus, the equilibrium 
parameters can be considered as uniform over a distance $\Delta y<c_{s} T_{0}$. The stability analysis, carried out for a uniform semi-infinite medium, can be applied to perturbations with sufficiently short wavelength $k \Delta y>1$. It follows that a necessary condition for the validity of the stability model is $k c_{s} T_{0}>>1$. For such wavelengths, Eq. (17) can be used in Eq. (14) to derive the function $\xi(t)$. Thus, Eq. (14) can be written in the following form:

$$
\frac{d^{2} \xi}{d t^{2}}-\gamma_{c}^{2}\left[1+q \sin \left(\omega_{0} t+\phi\right)\right] \xi=0
$$

where $\gamma_{c}=\sqrt{A\left|k g_{0}\right|}$ is the classical growth rate, $q=\sqrt{\alpha^{2}+9 \epsilon^{2} / 4}$, and $\phi=\tan ^{-1}(3 \in / 2 \alpha)$. Notice that Eq. (18) is a Mathieu equation, whose solution has the form $\xi(t)=\sigma(t) \exp (\mu t)$, with $\sigma(t)$ being periodic with period $\omega_{0}$. Using Eq. (13), the growth rate of the instability can be easily derived,

$$
\gamma=-k \beta \frac{1}{T_{0}} \int_{0}^{T_{0}} V_{a}\left(t^{\prime}\right) d t^{\prime}+\mu
$$

where $\beta=1.5$ for the simplified stability model. However, when Eq. (19) is compared to the Takabe's formula, we let $\beta=\beta^{T}=3-4$. In order to find $\mu$, one needs to numerically solve Eq. (18) for one period of oscillation. Figure 1 shows the parameter $\mu$, plotted versus the wave number $k$, for the following equilibrium parameters $d=20 \mu \mathrm{m}, g_{0}=5 \times$ $10^{15} \mathrm{~cm} / \mathrm{s}^{2}, A=1,\left\langle V_{a}\right\rangle=7 \times 10^{4} \mathrm{~cm} / \mathrm{s}, c_{s}=10^{6} \mathrm{~cm} / \mathrm{s}, T_{0}=0.3 \times 10^{-9} \mathrm{~s}, \phi=0$, and $q=0-2.5-3.5$. The validity of the stability model requires $\lambda=2 \pi / k<<20 \mu \mathrm{m}$. For any value of $q$ and $\omega_{0}$, it is possible to identify intervals of the $k$ axis where $\operatorname{Re}[\mu]=0$. We denote such intervals as dynamically stabilized (DS) regions, and we emphasize the importance of ablative convection [see Eq. (19)] at shorter wavelengths. According to Eqs. (1) and (19), the short wavelength modes are stabilized by convection and the cut-off wave number is $k_{c}=g A / \beta^{2} V_{A}^{2}$. It follows that an efficient dynamic stabilization can be achieved by choosing values of $q$ and $\omega_{0}$ that cause the first DS region to be located inside the interval $0<k<k_{c}$. In Fig. 2, the growth rates derived from Eq. (19) for $q=$ $0-2.5-3.5$ and $\beta=3.5$ (as given by Takabe $e$ t al. ${ }^{3}$ ) are shown. Observe that as $q$ increases, a better stabilization is induced at longer wavelengths, but shorter wavelengths can be destabilized $(q=3.5)$. This short wavelength instability is driven by the oscillations in the acceleration, with the perturbation having the characteristic structure of an oscillatory mode with exponentially increasing amplitude. For convenience, we denote these short wavelength modes as "parametric instabilities." Furthermore, when the mode 
wavelength is smaller than the density gradient scale length $\left[\delta=|(1 / \rho) d \rho / d y|^{-1}\right]$, the sharp boundary model is not valid and Eq. (19) cannot be used.

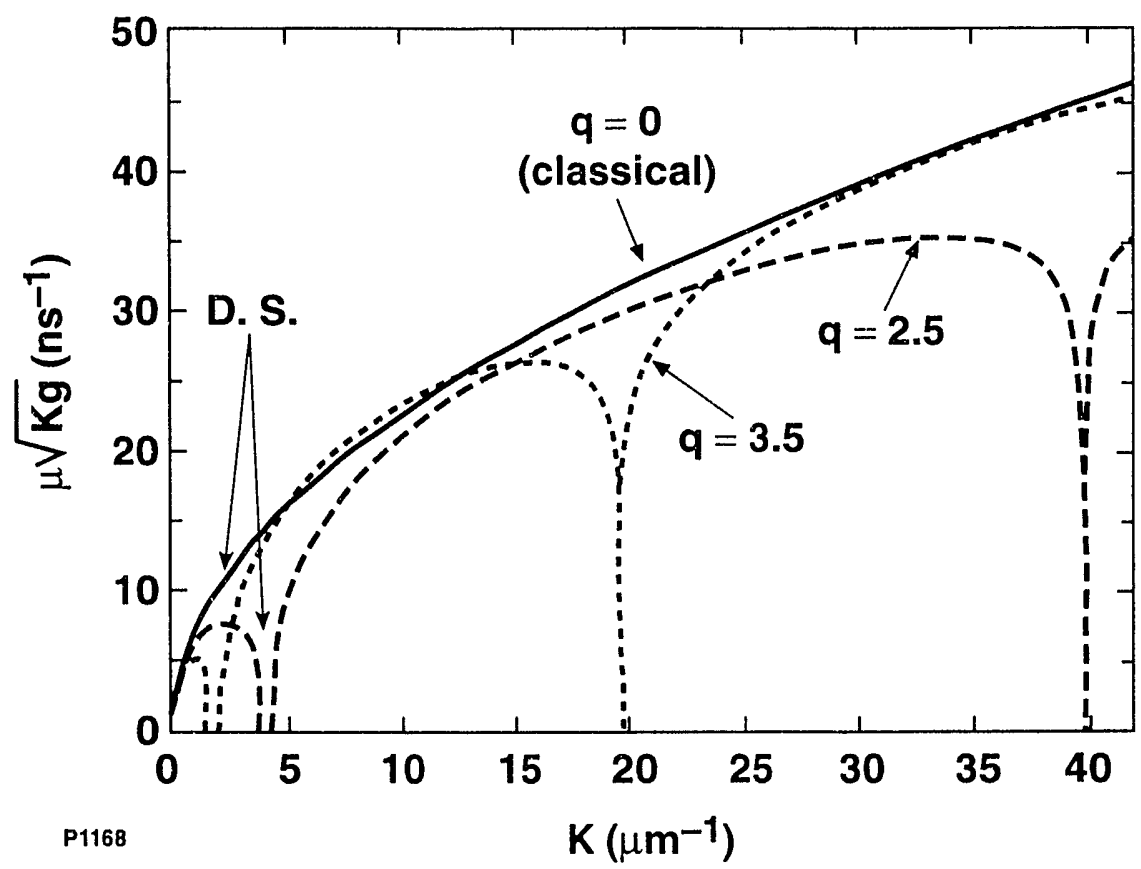

Fig. 1 Plot of the instability drive term $\mu$, versus the mode wave number $k$ for modulated $(q \neq 0)$ and unmodulated $(q=0)$ laser intensity.

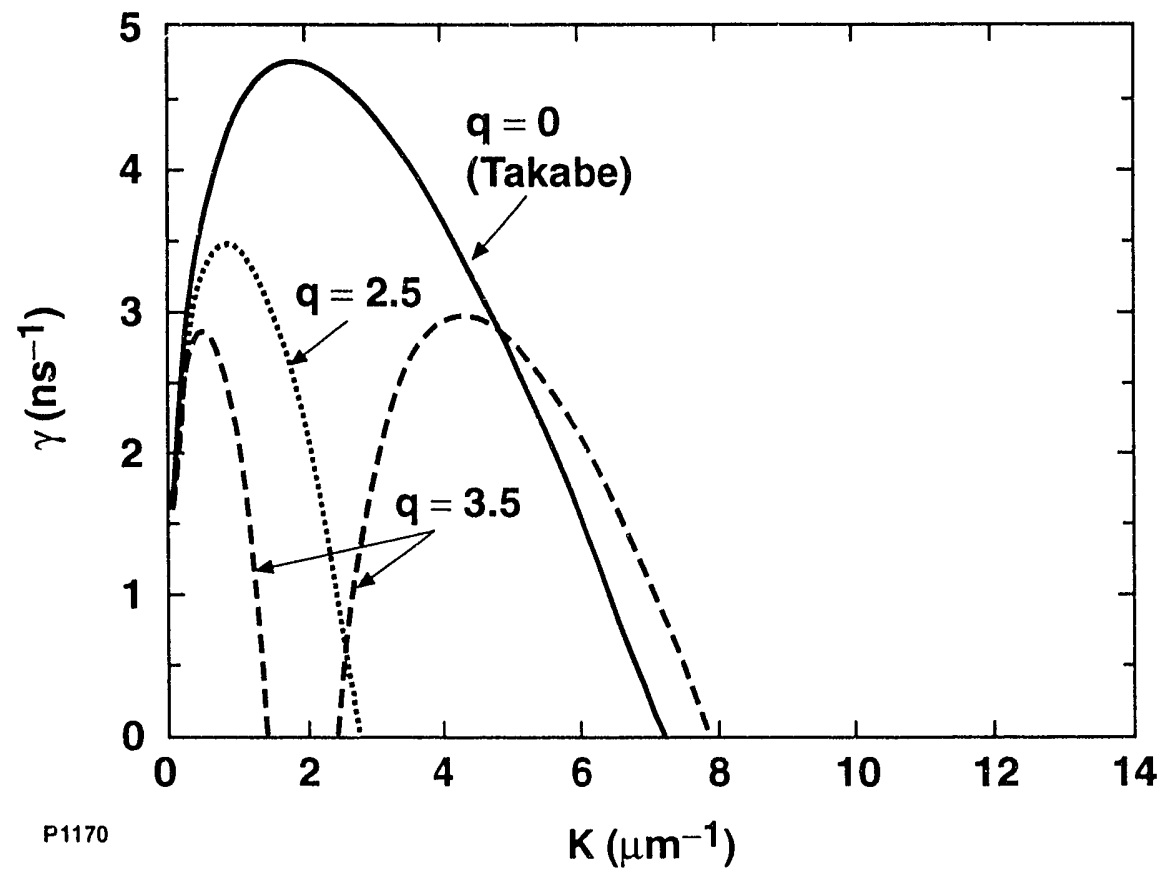

Fig. 2 Plot of the instability growth rate versus the mode wave number $k$ for modulated $(q \neq 0)$ and unmodulated $(q=0)$ laser intensity. 
The results of the analytic theory have been compared with the two-dimensional simulations obtained using the code $O R C H I D .{ }^{7}$ We have considered an $18-\mu \mathrm{m} \mathrm{CH}$ planar target, irradiated by a uniform laser beam. The laser intensity is modulated in time with a period of 0.3 ns. The modulation amplitude is $100 \%$, and the flat-top average intensity is $50 \mathrm{TW}$. For an accurate comparison with the analytic stability theory, we derive the equilibrium parameters $g,\left\langle V_{a}\right\rangle$ and $q$, from the one-dimensional code LILAC. ${ }^{8}$ The result is $g=4.5 \cdot 10^{15} \mathrm{~cm} / \mathrm{s}^{2},\left\langle V_{a}\right\rangle=7 \cdot 10^{4} \mathrm{~cm} / \mathrm{s}^{2}, \delta=1.5-2 \mu \mathrm{m}, \phi=0$, and $q=3.5-5.5$. In the two-dimensional simulation, an initial single wavelength perturbation evolves for 3 ns. Figure 3 shows a comparison between the linear growth rate derived from the simulation, with the one given by Eq. (19). Three regions of the $k$-axis can be identified: (1) The long wavelength region with $k<0.2 \mu \mathrm{m}^{-1}$, where the growth rate is virtually insensitive to the modulation of the laser intensity and very close to the classical value. (2) The intermediate wavelength region with $0.2<k<1$. For these values of the wave number, the dynamic stabilization is particularly effective. Observe that for $\lambda=2 \pi / k \approx 7 \mu \mathrm{m}$, the mode is completely stabilized. (3) The short wavelength region is defined as having a wave number $k>1$. In this region $k \delta>1$ and the effect of finite density-gradient scale length cannot be neglected. Notice that the simulation shows the presence of an unstable mode with wavelength $\lambda \approx 5 \mu \mathrm{m}$. Using Eq. (19) beyond its limit of validity $(k \delta<1)$ and dividing $\gamma_{c}^{2}$ by $(1+\alpha k \delta)$ with $\alpha<1$, we would predict the existence of parametric instabilities at shorter wavelengths (Fig. 3). However, the structure of the perturbation observed in the numerical simulation does not clearly show the characteristics of a parametric instability. Furthermore, the cut-off wave number observed in the numerical simulation (with or without laser intensity modulation) is much shorter than the one predicted by Eqs. (1) and (19). The stability of very short wavelength perturbations needs further investigation to determine an accurate value of the cutoff wave number.

The dynamic stabilization of the Rayleigh-Taylor instability in ICF targets was first observed in the numerical simulations by J. Boris. ${ }^{9}$ At that time, the ablative stabilization was still relatively unknown and a self-consistent theory for the stability of unsteady equilibria could not be derived. In this Letter we have shown the derivation of the linear stability theory of unsteady ablation fronts, and the conditions for the dynamic stabilization of the ablative Rayleigh-Taylor instability. The growth rate of the instability has been calculated for a sinusoidal modulation of the laser intensity. It is shown that an appropriate modulation frequency and amplitude can stabilize a large portion of the unstable spectrum and significantly reduce the maximum growth rate. 


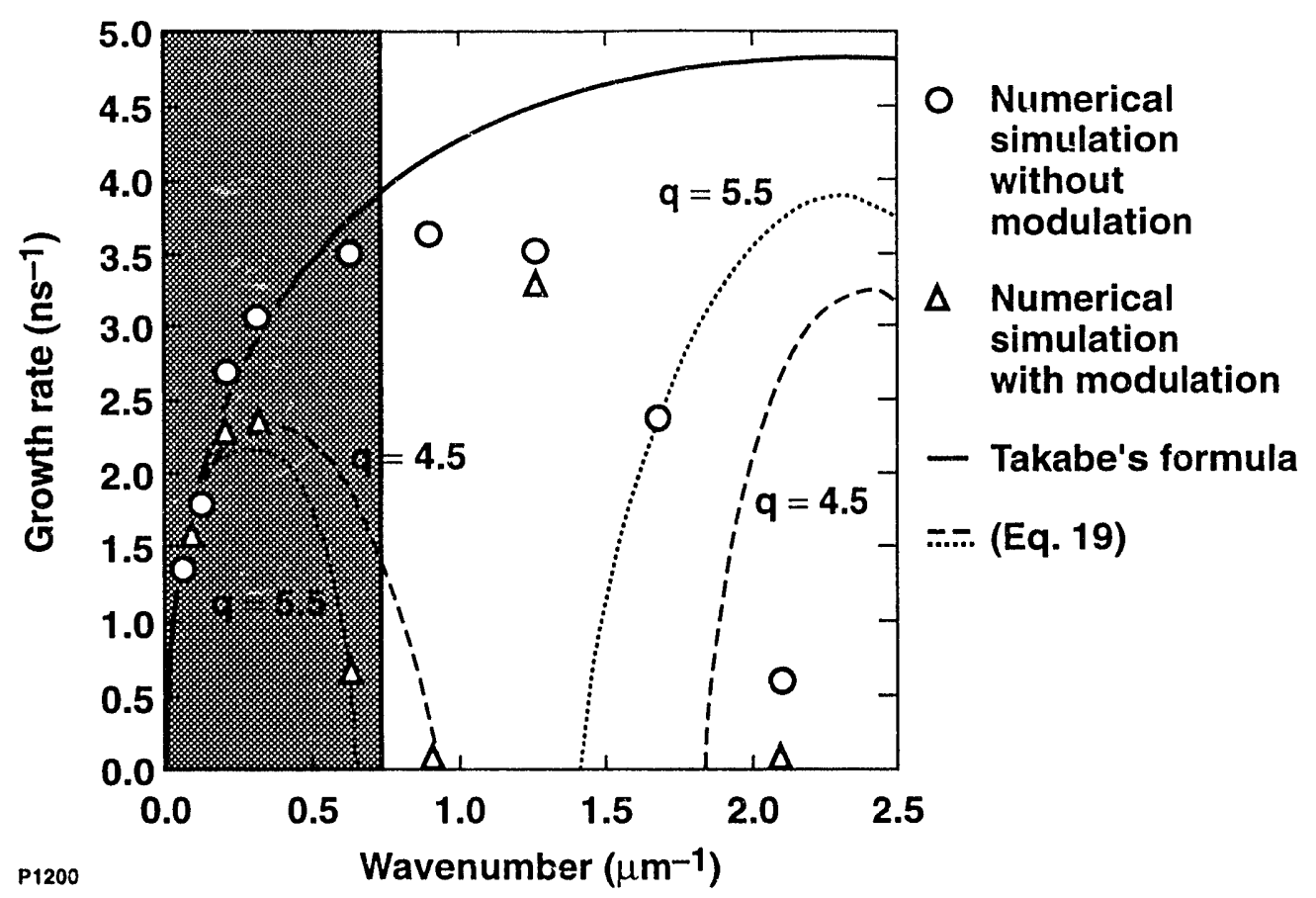

Fig. 3 Comparison of the growth rate obtained from numerical simulations (with modulation $\Delta$ and without modulation O) and the modified Eq. (19) with $\alpha=0.2, q=4.5$ (dashed), $q=5.5$ (dotted). The solid line represents Takabe formula and the shaded area represents the region with $\kappa \delta<1$.

\section{ACKNOWLEDGMENTS}

The authors would like to thank Prof. Al Simon for useful discussions. This work was supported by the U.S. Department of Energy Office of Inertial Confinement Fusion under Cooperative Agreement No. DE-FC03-92SF19460, the University of Rochester, and the New York State Energy Research and Development Authority. The support of DOE does not constitute an endorsement by DOE of the views expressed in this article.

\section{REFERENCES}

1. Lord Rayleigh, Scientific Papers (Cambridge University Press, Cambridge, England, 1900), Vol. II, p. 200.

2. S. Bodner, Phys. Rev. Lett. 33, 761 (1974).

3. H. Takabe, K. Mima, L. Montierth, and R. L. Morse, Phys. Fluids 28, 3676 (1985).

4. H. J. Kull and S. I. Anisimov, Phys. Fluids 29, 2067 (1986).

5. A. B. Bud'ko arid M. A. Liberman, Phys. Rev. Lett. 68, 178 (1992).

6. N. Rostoker and H. Tahsiri, Comments Plasma Phys. Cont. Fusion 3, 39 (1977).

7. R. L. McCrory and C. P. Verdon, Computer Applications in Plasma Science and Engineering, edited by Adam T. Drobot (Springer-Verlag, NY, 1991), pp. 291-314.

8. E. Goldman, Laboratory for Laser Energetics Report No. 16 (1973); Report No. 36 (1976).

9. J. P. Boris, Comments Plasma Phys. Cont. Fusion 3, 1 (1977). 

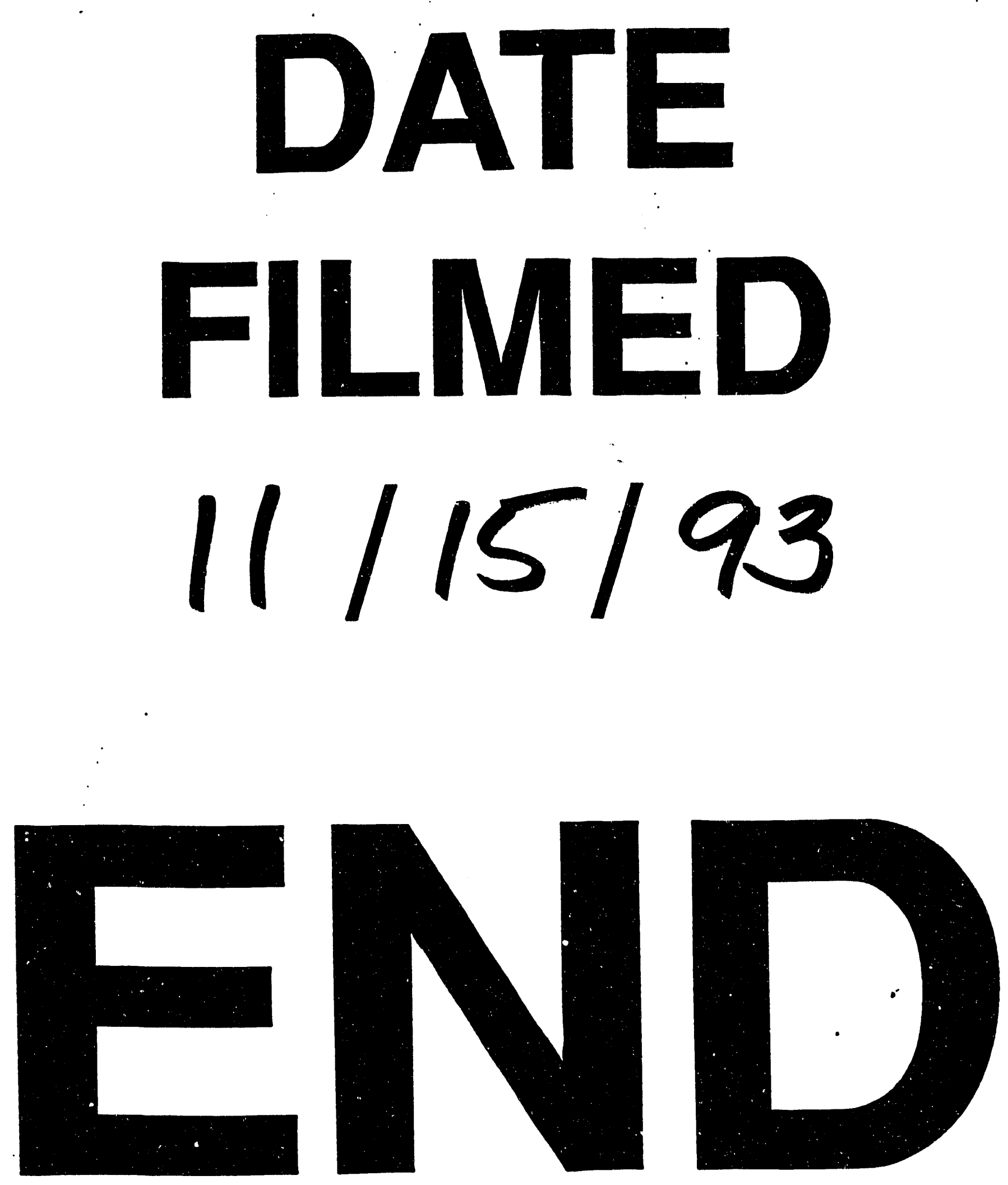
